\title{
AN OVERVIEW OF MIKE MORAVCSIK'S PUBLICATION ACTIVITY IN PHYSICS
}

\author{
R. TODOROV,* M. WINTERHAGER** \\ "Centre for Science Studies Bulgarian Academy of Sciences BG-1000 Sofia, Serdika St No 4 (Bulgaria) \\ **Science Studies Unit, University of Bielefeld, D-4800 Bielefeld 1 (FRG)
}

(Received December 19, 1989)

\begin{abstract}
A bibliometric online technique is applied on data from the INSPEC bibliographic file to describe some aspects of Moravcsik's publication activity (co-authorship, source journals, etc.). Separately, a co-occurrence method is used to represent the subject structure (the main topics and their links) of his papers in physics. The principle underlying this method is to develop a network based on common appearances of classification subdivisions (headings) as well as of controlled terms in Moravcsik's document records. The results, in the form of line and point graphs, give a global picture of Mike Moravcsik's research profile in physics.
\end{abstract}

\section{Introduction}

Host systems for scientific and technical information are increasingly used for bibliometric purposes, 1,2 namely, data gathering (creating appropriate subsets of document records) and internal processing (arranging and combining of a large number of bibliographic elements). Accessible data have contributed to introduction of advanced quantitative methods (analyses of co-occurrences ${ }^{3-5}$ and multivariate techniques ${ }^{6,7}$ ) to describe cognitive and/or social structures of science and technology (sub)fields for different purposes. ${ }^{8,9}$ These methods are based mainly on the absolute and common appearance of preselected bibliographic elements (such as citations, controlled and supplementary terms, title words, headings, etc.) in document records. More specifically, co-appearances are used to construct a network in which relations between elements are traced according to a given similarity measure (simple co-occurrence, inclusion index, proximity index, Jaccard index, strength index, etc.). ${ }^{10}$ References to such studies are given in recent articles and conference papers. ${ }^{11-14}$

The purpose of this paper is to describe some aspects of Moravcsik's papers in physics and to display their structure by applying a bibliometric approach (online technique and a co-occurrence method).

\footnotetext{
‡ Dedicated to the memory of Michael J. Moravcsik
} 


\section{Data and method}

Data for the analysis have been obtained by logging on the INSPEC file. An INSPEC record includes traditional bibliographic fields such as Title (TI), Author (AU), Source (SO), Document Type (DT), and a set of elements expressing the subject content of a document: Classification Codes (CC), Treatment Codes (TC), Controlled Terms (CT) and Supplementary Terms (ST). CC stand for subject headings or topics which fall into the following categories: substances, properties, phenomena, theories, methods, models, instruments and etc.

For the purpose of the study all document records containing the name of M.(J). Moravcsik in the "Author" field have been downloaded from the INSPEC file. A total of 89 document records have been counted and downloaded. Six fields (elements) of this set of records have been selected for quantitative analysis: CC, DT, TC, AU (Author and Affiliation), CT and SO.

Online technique ${ }^{1}$ has been applied on the downloaded set of records to generate sorted lists of headings, controlled terms, co-authorship, affiliations, and source journals. Then, an appropriate software has been developed for constructing a triangular matrix $\mathrm{C}=\left\{\mathrm{C}_{\mathrm{ij}}\right\}$ containing the frequencies of appearance and coappearance of all headings (as well as Controlled Terms) $\mathrm{C}_{\mathrm{i}}$ that occur 3 or more times in the set of the 89 selected records. (Two headings $C_{i}$ and $C_{j}$ are said to cooccur if there is at least one record containing both $C_{i}$ and $C_{j}$ ) A diagonal element $C_{i i}$ is the absolute frequency of appearance of $C_{i}$ and an off-diagonal element $C_{i j}$ represents the co-occurrence of $\mathrm{C}_{\mathrm{i}}$ and $\mathrm{C}_{\mathrm{j}}$ in the set of records under consideration.

The matrix $C=\left\{C_{i j}\right\}$ is further used for calculating an inclusion index $I_{i j}=C_{i j} /$ $\min \left(C_{\mathrm{ii}}, \mathrm{C}_{\mathrm{jj}}\right)$ which emphasizes the "central poles" or foci of Moravcsik's research profile and reveals their relations with other "peripheral" topics. 15 The $I_{i j}$ expresses the degree to which two headings are related. It is a measure of similarity and is not symmetrical: the link between $C_{i}$ and $C_{j}$ could be drawn as an arrow from $C_{j}$ (lower frequency of occurrence) to $C_{i}$ (higher frequency of occurrence). High values of $I_{i j}$ are assumed to mean close links between headings (or Controlled Terms). If a horizontal scale is introduced for the frequency of appearance a graph is obtained for the set under consideration: the covered subject headings (which occur and co-occur above selected threshold values) are displayed as points on a map with their links. (Vertically, headings are arranged in such a way that the number of intersections between connecting arrows is minimized.) Thus, distances between headings do not indicate the strength of links. 


\section{Results}

Lists of headings and controlled terms which appear most frequently in Moravcsik's document records (INSPEC file) are given in Tables 1-2. In Table 3 the names of Moravcsik's co-authors are shown. All universities quoted in the "Author" field of his publications are ranked in decreasing order of appearance in Table 4, while the journal titles arranged according to the number of published articles are presented in Table 5.

Table 1

Most frequently appearing headings in Moravcsik's document records

\begin{tabular}{rll}
\hline$F^{*}$ & CC & \multicolumn{1}{c}{ Headings } \\
\hline 29 & A1388 & Polarisation in interactions and scattering \\
20 & A1375C & Nucleon-nucleon interactions (E < 10GeV) \\
12 & A1130E & Charge conjugation, parity, time reversal and other discrete symmetries \\
8 & A2470 & Polarisation in reactions and scattering \\
6 & A1385D & Elastic scattering $(\mathrm{E}>10 \mathrm{GeV})$ \\
5 & A1010 & Models of weak interactions \\
4 & A1240R & Peripheral models \\
4 & A1290 & Miscellaneous theoretical ideas and models \\
4 & A1420P & Dibaryons \\
4 & A2140 & Few-nucleon systems \\
4 & A2410 & Nuclear reaction and scattering models \\
4 & A2710 & Nuclei with mass number 1 to 5 \\
3 & A1130C & Lorentz and Poincare invariance \\
3 & A1235E & Application of QCD to particle properties and reactions \\
3 & A1240 & Models of strong interactions \\
3 & A1375G & Pion-baryon interactions (E < 10 GeV) \\
3 & A1385 & Hadron-induced interactions (E $>10 \mathrm{GeV}$ ) \\
3 & A2130 & Nuclear forces \\
2 & & 10 headings \\
1 & & 60 headings \\
\hline
\end{tabular}

F: Frequency of appearance. 


\section{R. TODOROV, M. WINTERHAGER: MORAVCSIK'S PUBLICATION ACTIVITY}

Table 2

Most frequently appearing Controlled Terms

\begin{tabular}{rl}
\hline$F^{*}$ & \multicolumn{1}{c}{ Controlled Terms } \\
\hline 19 & Polarisation in elementary particle scattering \\
16 & Polarisation in elementary particle interactions \\
16 & Proton-proton scattering \\
10 & Polarisation in nuclear reactions and scattering \\
7 & Elementary particle interactions \\
6 & T invariance \\
6 & Helicity (Elementary particles) \\
6 & P invariance \\
5 & Pion production \\
5 & Baryon resonances \\
5 & Elementary particle interaction models \\
5 & Nuclei with number 1 to 5 \\
4 & Wave functions \\
4 & Colour model \\
4 & Elementary particle theory \\
4 & Many-body problems \\
4 & Nuclear forces \\
4 & Nuclear reaction and scattering theory \\
4 & Peripheral models \\
3 & Conservation laws \\
3 & Deuterons \\
3 & Elementary particle strong interactions \\
3 & Nucleon-nucleon interactions \\
3 & Polarisation \\
3 & Proton-proton interactions \\
3 & Quantum theory \\
2 & 9 Controlled Terms \\
1 & 53 Controlled Terms \\
\hline
\end{tabular}

F: Frequency of appearance.

Table 3

Co-authorship in 89 Moravcsik's paper

\begin{aligned} & \hline F* $^{*} \multicolumn{1}{c}{$ Author } \\ & \hline 89 Moravesik, M.J. \\ & 46 Goldstein, G.R. \\ & 11 Arash, F. \\ & 3 Ghahramany, N. \\ & 3 Kushno, D. \\ & 2 Certov, A. \\ & 2 Kiwon Nam \\ & 2 Mathelitsch, L. \\ & 2 Murugesan, P. \\ & 2 Reid, R.V., Jr. \\ & 2 Wing-Yin Yu \\ & 1 Other 20 co-authors \\ & \hline\end{aligned}

F: Frequency of appearance. 
Table 4

Moravcsik's affiliations

\begin{aligned} & \hline F* $^{*}$ University \\ & \hline 44 Univ. of Oregon, Eugene, OR, USA \\ & 30 Tufts Univ., Medfrod, MA, USA \\ & 4 Graz Univ., Austria \\ & 3 Univ. California, Davis, USA \\ & 1 Osaka Univ., Toyonaka, Japan \\ & 1 Oxford Univ., UK \\ & 1 Triumf, Vancouver, British Columbia, Canada \\ & 1 Univ. Michigan, Ann Arbor, USA \\ & 1 Univ. of Indonesia, Jakarta, Indonesia \\ & 1 Univ. of Sussex, Brighton, England \\ & \hline\end{aligned}

F: Frequency of appearance.

Table 5

Distribution of Moravesik's articles over Journals Titles

\begin{aligned} & \hline$N^{*} \multicolumn{1}{c}{$ Journal Title (Country) } \\ & \hline 26 Phys. Rev. D, Part. Fields (USA) \\ & 9 Phys. Rev. Lett. (USA) \\ & 5 Ann. Phys. (USA) \\ & 5 Phys. Lett. B (Netherlands) \\ & 5 Phys. Rev. C, Nucl. Phys. (USA) \\ & 4 J. Math. Phys. (USA) \\ & 3 J. Phys. Colloq. (France) \\ & 2 Int. J. Mod. Phys. A (Singapore) \\ & 2 Lett. Nuovo Cimento (Italy) \\ & 2 Nucl. Instrum. \& Methods A (Netherlands) \\ & 2 Nucl. Phys. B (Netherlands) \\ & 2 Nuovo Cimento A (Italy) \\ & 2 Phys. Rev. (USA) \\ & 2 Phys. Today (USA) \\ & 2 Scientometrics (Netherlands) \\ & 1 Other 13 journal titles \\ & \hline\end{aligned}

N: Number of articles.

The subject profile of Moravcsik's activity in physics (using correspondingly headings and controlled term) is displayed on Figs 1 and 2. Positions are obtained by ordering elements horizontally by increasing frequency of occurrence. The lines are drawn from topics with lower frequency of appearance to such with higher one. A threshold $\mathrm{I}_{\mathrm{o}}=0.4$ is introduced and inclusion index values are indicated correspondingly by solid and dotted lines. 

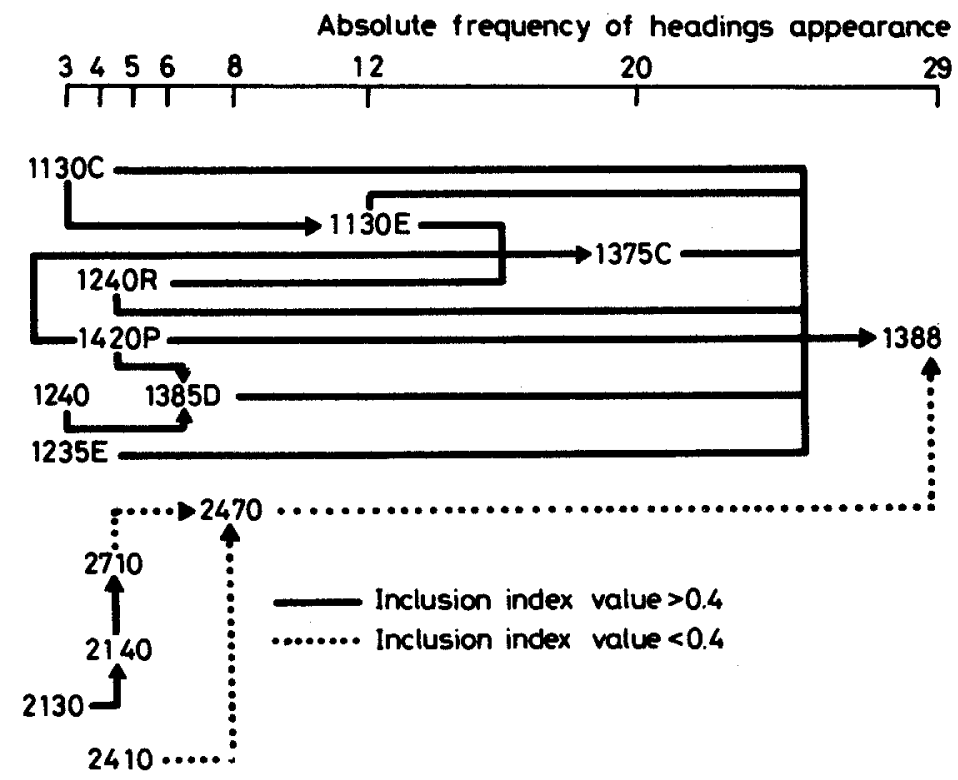

Fig. 1. Map of Headings for Moravesik's profile as a physicist.

$1130 \mathrm{C}$ Lorentz and Poincare invariance

1130E Charge conjugation, parity, time reversal and other discrete symmetries 1235E Application of QCD to particle properties and reactions

1240 Models of strong interactions

1240R Peripheral models

1375C Nucleon-nucleon interaction $(E<10 \mathrm{GeV})$

1385D Elastic scattering $(E>10 \mathrm{GeV}$ )

1388 Polarisation in interactions and scattering

1420P Dibaryons

2130 Nuclear forces

2140 Few-nucleon systems

2410 Nuclear reaction and scattering models

2470 Polarisation in reactions and scattering

2710 Nuclei with mass number 1 to 5 


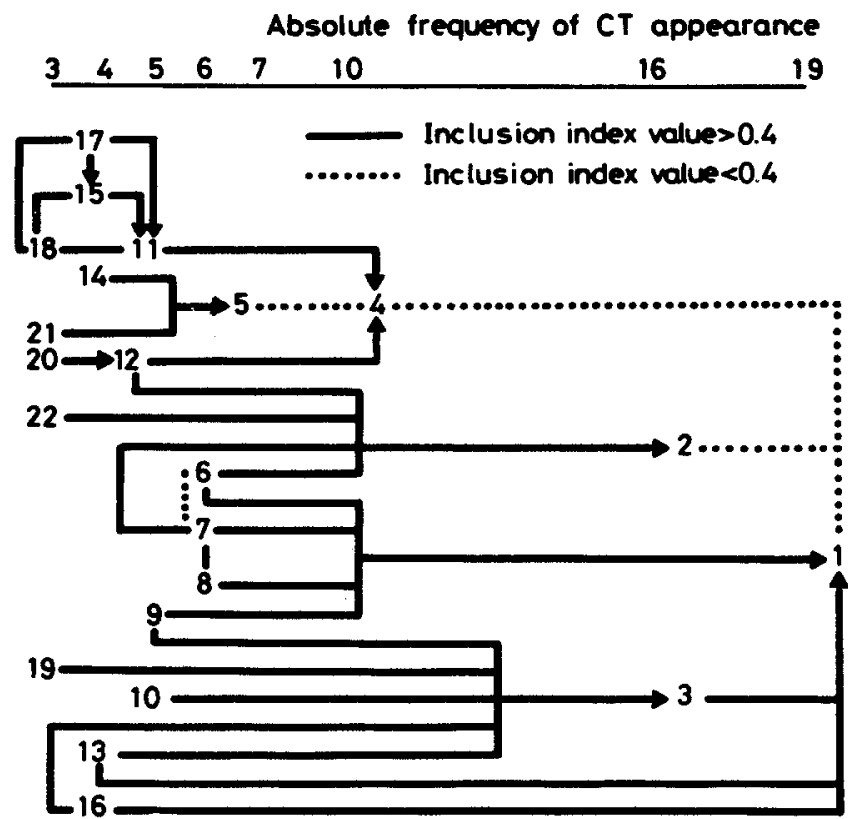

Fig. 2. Map of Controlled Terms (CT) for Moravesik's profile as a physicist.

1. Polarisation in elementary particle scattering;

2. Polarisation in elementary particle interactions;

3. Proton-proton scattering;

4. Polarisation in nuclear reactions and scattering;

5. Elementary particle interactions;

6. Helicity (elementary particles);

7. $P$ invariance;

8. $T$ invariance;

9. Baryon resonances;

10. Elementary particle interaction models;

11. Nuclei with mass number 1 to 5 ;

12. Pion production;

13. Colour model;

14. Elementary particle theory;

15. Nuclear forces;

16. Peripheral models;

17. Wave functions;

18. Deuterons;

19. Elementary particle strong interactions;

20. Nucleon interactions;

21. Polarisation;

22. Proton-proton interactions.

\section{Discussion}




\section{R. TODOROV, M. WINTERHAGER: MORAVCSIK'S PUBLICATION ACTIVITY}

In this paper online technique and a mapping method are used to give an overview of Moravcsik's publication activity in physics. It is not the purpose of the study to discuss conceptual issues of the profile obtained or to justify the selection of record elements.

The description of some aspects of Moravcsik's papers in physics as well as the displaying of their reduced (content) structure have been done rather to demonstrate potential of online bibliometrics and to show some of the capacities of a cooccurrence method to unveil (relatively easy) an individual research profile without consulting experts in the corresponding field. It appears, for example, that Moravcsik's papers in physics are mainly theoretical (83\%) and published predominantly in journals. Half of the articles are published only in three journals (see Table 5). This could be explained by the subject characteristics of Moravcsik's specialty (narrow and rather self-contained). All the journals are highly visible not only in the field of elementary particle physics. More than $80 \%$ of Moravcsik's papers have been prepared at the University of Oregon and Tufts University (see Table 4). As main co-authors in Mike Moravcsik's papers appear Goldstein G.R. and Arash F. (see Table 3). Tables 3 to 5 have been generated online by logging on the INSPEC file. Headings and Controlled Terms which appear most frequently in Moravcsik's document records are listed in Tables 1 and 2. The Concordance to the INSPEC Classification has been used to take account of changes that have occurred during the period under consideration. Headings and Controlled Terms from Moravcsik's papers preserve their names and definitions. In this way an additional agreement of terms has appeared superfluous and co-occurrence method could be applied directly.

The maps on Figs 1 and 2 show similarities and differences in detailed subject coverage of Moravcsik's activity in physics. Both maps present a monopolar structure. The central pole of the "heading" map (Fig. 1) is "Polarisation in elementary particle interactions and scattering". It includes two of the most frequently used controlled terms (see Table 2). The "heading" map is dominated by the part of elementary particle physics (EPP). The group linked to $1375 \mathrm{C}$ (Nucleonnucleon interactions) is prevailing on the map and represents a rather strongly connected intermediary network (see Fig. 1). It includes as a mediator "Charge conjugation, parity, time reversal and other discrete symmetries" from a larger EPP topic (Symmetry and conservation laws). The nuclear physics (NP) aspects of polarisation in interactions and scattering appear rather as marginal. "Elastic scattering" emerges as a peripheral topic which is strongly connected to the central pole. 
On Figure 2 "polarisation in interactions" is rather separated from "polarisation in scattering". The topic "proton-proton scattering" and its modeling have gained an important position. NP issues related to "polarisation in nuclear reactions and scattering" appear as a separated group which includes several peripheral topics. In other words, Fig. 2 reveals more details as compared to the network based on headings.

\section{Conclusion}

Some aspects of Moravcsik's publication activity in physics are directly described by using online bibliometrics. The capacities of a co-occurrence method are demonstrated for representing his individual research profile or problem area: all Moravcsik's papers have been reduced to a set of linked headings (and separately controlled terms). The obtained structure is dominated by several strongly linked research topics which are easily subdivided in peripheral, intermediary, and central. Regardless of the time differences in publication, Moravcsik's papers in physics could be reduced to a small number of interlinked topics with determined positions. Thus, his publication activity could be characterized as concentrated, continuous (minor changes in objects and phenomena of research), and progressive (variation in the proposed solutions).

\section{References}

1. O. Persson, Measuring Scientific Output by Online Techniques, In: A.F.J. VAN RaAn (Ed.), Handbook of Quantitative Studies of Science and Technology, North-Holland, Amsterdam, 1988, pp. 229-252.

2. R. Todorov, Representing a scientific field: A bibliometric approach, Scientometrics, 15 (1989)589601.

3. H. SMALL, E. GARFIELD, The geography of science: Disciplinary and national mappings, Joumal of Information Science, 11 (1985) 147-159.

4. W.L. GuIST, L. GeORGHIOU, The use of co-nomination analysis in real-time evaluation, Scientometrics, 14 (1988) 265-281.

5. J.-P. COURTLAL, J. LAw, A co-word study of artificial intelligence, Social Studies of Science, 19 (1989) 301-311.

6. R.J.W. TusSEN, J. De LeEuw, Muitivariate data-analysis methods in bibliometric studies of science and technology, In: A.F.J. VAN RAAN (Ed.), Handbook of Quantitative Studies of Science and Technology, North-Holland, Amsterdam, 1988, pp. 705-740.

7. R. TODOROv, M. WINTERHAGER, Mapping Australian, Geophysics: A co-reading analysis, Scientometrics, 18 (1990) 35.

8. P. Healey, H. Rothman, P.K. Hoch, An experiment in science mapping for research planning, Research Policy, 15 (1986) 233-251. 


\section{R. TODOROV, M. WINTERHAGER: MORAVCSIK'S PUBLICATION ACTIVTTY}

9. A. RIP, Mapping of science: Possibilities and limitations, In: A.F.J. VAN RAAN (Ed.), Handbook of Quantitative Studies of Science and Technology, North-Holland, Amsterdam, 1988, pp. 253-273.

10. J.-P. CourTial, Technical issues and developments in methodology, In: M. CaLLON, J. LAW, A. RIP (Eds), Mapping the Dynamics of Science and Technology, Mac-Millan, London, 1986, pp. 189-193.

11. J.J. FrankIIN, R. JohNSton, Co-citation bibliometric modeling as a tool for S\&T policy and R\&D management: Issues, applications, and developments, In: A.F.J. VAN RAAN (Ed.), Handbook of Quantitative Studies of Science and Technology, North-Holland, Amsterdam, 1988, pp. 325-389.

12. J.E.J. OBERSKI, Some statistical aspects of co-citation cluster analysis and a judgement by physicists, In: A.F.J. Van RAAN (Ed., Handbook of Quantitative Studies of Science and Technology, NorthHolland, Amsterdam, 1988, pp. 431-462.

13. R.R. BRAAM, H.F. MoED, A.F.J. VAN RAAN, Comparison and combination of co-citation and coword clustering, In: A.F.J. VAN RAAN, A.J. NEDERHOF, H.F. MOED (Eds), Science and Technology Indicators, DSWO Press, University of Leiden, 1989, pp. 307-337.

14. R. TODORov, Co-classification analysis for science mapping : An example from superconductivity, In: A.F.J. VAN RAAN, A.J. NeDeRHof, H.F. MOED (Eds), Science and Technology Indicators, DSWO Press, University of Leiden, 1989, pp. 261-270.

15. M. Callon, J. Law, A. RIP Qualitative scientometries, In: M.Callon, J. LAw, A. RIP (Eds), Mapping the Dynamics of Science and Technology, Macmillan, London, 1986, pp. 103-123. 